\title{
Size dependent magnetic and dielectric properties of $\mathrm{Sm}_{2} \mathrm{CoMnO}_{6}$
}

\author{
Alo Dutta ${ }^{1 *}$, P. K. Mukhopadhyay ${ }^{1}$, Sadhan Chanda ${ }^{2}$ and T. P. Sinha ${ }^{2}$ \\ ${ }^{1}$ Department of Condensed Matter Physics and Material Sciences, \\ S. N. Bose National Centre for Basic Sciences, \\ Block-JD, Sector-III, Salt Lake, Kolkata-700 106, India \\ ${ }^{2}$ Department of Physics, Bose Institute, 93/1, A. P. C. Road, Kolkata-700009, India \\ *Email: alo_dutta@yahoo.com
}

Recently the effect of size reduction on the crystallographic phase stability and ground state property, which has therefore effected the change in the physical properties of rare earth cobaltites and manganites, has gained much research interest [1]. In relation to this, we have investigated the size dependent magnetic and dielectric properties of a double perovskite oxide $\mathrm{Sm}_{2} \mathrm{CoMnO}_{6}$ (SCMO) synthesized by sol-gel citrate process. The calcination temperature of nano SCMO (SCMO-n) and bulk SCMO (SCMO-b) is $800{ }^{\circ} \mathrm{C}$ and $1200{ }^{\circ} \mathrm{C}$ and corresponding particle size is found to be $106 \mathrm{~nm}$ and $1.2 \mu \mathrm{m}$ respectively. The Rietveld refinement of the XRD profile suggests that SCMO-n has orthorhombic Pnma phase whereas SCMO-b has monoclinic $\mathrm{P} 21 / \mathrm{n}$ symmetry.

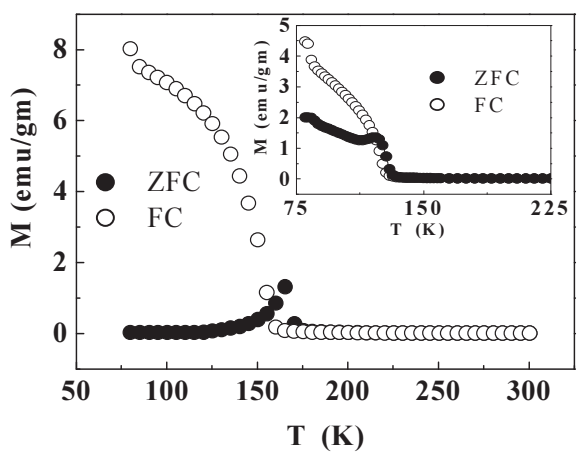

Figure 1: Temperature dependence magnetization of SCMO-n and SCMO-b (inset).

The temperature dependent FC (field cooled) and ZFC (zero field cooled) magnetization data taken at an applied magnetic field of $100 \mathrm{Oe}$ as shown in Figure 1 reveal the decrease of Curie temperature to $122 \mathrm{~K}$ for SCMO-b with respect to $161 \mathrm{~K}$ for SCMO-n which may be due to the lowering of crystallographic order. The increase of ZFC data for SCMO-b below the transition temperature may indicate the presence of another low temperature magnetic transition as observed in $\mathrm{La}_{2} \mathrm{CoMnO}_{6}$ [2].
Figure 2 shows the M-H curve of SCMO-n at different temperatures. The $\mathrm{M}-\mathrm{H}$ curve indicates the ferromagnetic behaviour with unsaturated magnetization below the transition temperature. At $300 \mathrm{~K}$ (above the transition temperature) M-H curve is linear corresponding to paramagnetic behaviour of the sample.

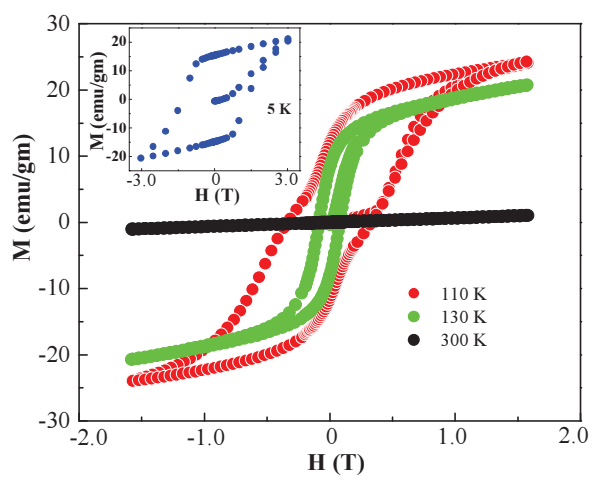

Figure 2: M-H curve of SCMO-n at different temperatures. Inset is the $\mathrm{M}-\mathrm{H}$ curve at $5 \mathrm{~K}$ upto 3.5 Tesla field.

The dielectric properties of the materials are investigated in the frequency range from $50 \mathrm{~Hz}$ to $1 \mathrm{MHz}$ and in the temperature range from $193 \mathrm{~K}$ to $373 \mathrm{~K}$ using alternating current impedance spectroscopy. For both the materials, ac conductivity follows the power law behaviour. The values of ac conductivity for SCMO-b is higher than SCMO-n. The mobility of charge carries decrease due to the scattering in the high-density interfacial states of nano-material and hence the value of ac conductivity is decreased with the decrease of particle size.

\section{References}

1. R. Nechache, C. Harnagea, F. Rosei, Adv. Mater. 23 (2011) 1724.

2. J.B. Goodenough, Magnetism and the chemical bond (New York: Inter Science, 1976). 